\title{
ECONOMIE ET INSTITUTIONS
}

Les Eaux et Forêts : du $12^{e}$ au $20^{e}$ siècle. Paris, Ed. du C.N.R.S., 1988. $16 \times 24$, 768 p., ill. (« Histoire de l'administration française »).

Réalisé par un collectif d'ingénieurs du Génie rural secondés par une archivistepaléographe, cet ouvrage embrasse une vaste synthèse historique, en étudiant les huit siècles d'efforts administratifs qui ont attiré le saltus et les eaux continentales au centre des politiques d'aménagement territorial. Le livre constitue une imposante somme de renseignements, traçant une fresque d'enchaînements et de ruptures dans un domaine où la connaissance restait fragmentaire.

Interstices entre les périmètres labourés, terres vaines, forêts, eaux dormantes et rivières échoient au ban seigneurial dès le $X^{\ell}$ siècle. En même temps, les usages communautaires qui grèvent les marges et les eaux sont renforcés. A partir du $\mathrm{xIr}^{\mathrm{e}}$ siècle cependant, le déploiement du domaine royal suscite la multiplication des agents forestiers de la couronne, désireuse d'accroître ses revenus domaniaux. L'autorité capétienne a aussi besoin de bois pour sa marine et sa poliorcétique. En 1291, Philippe le Bel confie la surveillance de ses bois à des maîtres enquêteurs des Eaux et Forêts. Ces derniers régissent une administration calquée sur le modèle seigneurial, avec des compétences interstratifiées, où se mêlent gruyers, prévôts et sergents. Pourtant, la gestion royale induit une pratique différente, avec des chocs en retour sur les châtellenies : mise en défens de secteurs réservés aux baliveaux, isolement de garennes, concession du droit de pêche à quelques habilités. Le domaine non labouré n'est plus exclusivement soumis à des activités prédatrices, il accède au rang de patrimoine renouvelable, mais au profit d'une élite nobiliaire.

Le $\mathrm{XVI}^{\ell}$ siècle accroît la confusion qui lie la protection des écrins ruraux et leur appropriation plus étroite. Pour renforcer leur emprise sur les Eaux et Forêts, François $I^{\text {er }}$ et Henri II recourent massivement aux officiers vénaux, sans maitriser l'explosion de leur effectif. Les unités de gestion forestière se trouvent ainsi fractionnées dans l'espace, mais aussi dans le temps, avec des relais d'autorité suivant les jours de la semaine. A côté de telles surcharges, les pans sous-évalués du domaine royal sont faiblement encadrés. En 1669, une ordonnance de Colbert simplifie cette prolifération brouillonne : elle supprime des offices inutiles, favorise la sauvegarde des futaies au détriment des usages, quadrille les forêts royales 
ou ecclésiastiques avec des commissaires réformateurs. Des normes de coupe et de balivage sont imposées aux bois des communautés et des particuliers. L'accès aux forêts domaniales et aux rivières navigables est juridiquement facilité, tandis que les droits de chasse et pêche sont réservés à un petit contingent de bénéficiaires.

Colbert ne se contente pas de légiférer, il multiplie les grands maîtres des Eaux et Forêts créés par Henri III, en les transformant en officiers vacataires. Sous Louis XV et Louis XVI, l'action forestière de ces agents supérieurs soutient la comparaison avec le zèle routier des Ponts-et-Chaussées. Le XvIrr siècle arrache pourtant à la monarchie des défrichements préjudiciables. A l'époque des Lumières, l'administration des Eaux et Forêts forme d'ailleurs un précipité qui ne parvient pas à acquérir les défenses corporatives dont se targuent les ingénieurs des Ponts-et-Chaussées. De ce fait, elle ne franchit pas indemne le cap de la Révolution. Menacés dès 1770 , les grands maîtres sont remplacés par des conservateurs en septembre 1791. Sous l'égide du conseiller d'État Bergon, les conservateurs impériaux se retrouvent à parité de considération avec les grands techniciens des Ponts-et-Chaussées. Malheureusement, sous la poussée libérale qui accompagne la déchéance de l'Empire, l'administration des Eaux et Forêts est fondue dans les services de l'enregistrement en 1817. Cet aboutissement marque le triomphe des égocentrismes que libère 1789 : initié à la facilité par la rétrocession des biens nationaux, l'État $\mathrm{du} \mathrm{XIX}^{\mathrm{e}}$ siècle s'engage dans la vente des forêts domaniales, que les marchands de biens et les acquéreurs dépècent à blanc.

Face aux risques écologiques que la France des notables fait courir à la collectivité, la tradition administrative ne tarde pas à réagir. Dès 1820, Louis XVIII rétablit une administration des Forêts autonome. En décembre 1824, Charles X fonde l'École royale forestière de Nancy, pour former l'encadrement supérieur du nouveau service. En 1827, l'ordonnance de 1669 est actualisée sous la forme d'un Code forestier. En 1873, l'École des Barres est ouverte dans le Loiret : elle complète l'établissement nancéien en instruisant les cadres intermédiaires. Immédiatement, les deux écoles militent pour le développement des futaies, à un moment où la houille détrône le fagot pour le chauffage, tandis que le bois de sciage est importé à plus de $50 \%$. La science sylvicole affermit ainsi son long combat contre les périmètres de coupes nettes.

En encourageant la gestion à long terme des forêts par leur aménagement, la nouvelle administration forestière se heurte aux habitudes des comptables du fisc, au corporatisme des maîtres de forge et des bûcherons, à l'avidité des spéculateurs. Ces groupes de pression se trouvent des chevaux de Troie, comme le directeur des Forêts Legrand, entre 1836 et 1851, ou le ministre de l'Agriculture Viette, en 1888-1889. C'est pourtant le choix du long terme qui porte au pinacle l'administration forestière du siècle dernier. Tandis que Nancy accueille des stagiaires venus de toute l'Europe, le Second Empire confie la restauration des terrains en montagne à ses forestiers d'État, en 1860 . A cette action contre le ravinement catastrophique, il ajoute le reboisement des Landes et la rationalisation des écosystèmes coloniaux. En 1898, l'administration des Forêts retrouve celle des Eaux, perdue en 1817. Dès lors, les techniciens des Eaux et Forêts s'érigent en maîtres jardiniers du cadre écologique.

A l'orée du $\mathrm{xx}^{\mathrm{e}}$ siècle, les Eaux et Forêts abordent l'ère des compétences 
ubiquitaires. Dès 1913, la loi Audiffred leur ouvre la gestion des forêts privées. En 1919 , les gardes des forêts communales sont domanialisés. Face à cette extension des prérogatives, les propriétaires privés tentent de protéger leur autonomie de gestion dans des associations de type 1901. En réalité, cette réaction engendre une synergie qui unit administration, regroupements de propriétaires et syndicats des métiers du bois. L'administration des Eaux et Forêts devient, en effet, la conseillère technique des partenaires qui structurent leur activité : elle en profite pour impulser la revitalisation des eaux et un reboisement ambitieux. Gênée entre les deux guerres par le manque de crédits, elle finit par s'ériger en cerveau de l'aménagement territorial. Tournée dès la Libération vers cette mission planificatrice par le ministre Leloup, l'administration des Eaux et Forêts est fusionnée avec le Génie rural, avec la réforme Pisani de 1965.

Dans le cadre de cette réorganisation, l'école de Nancy devient une annexe forestière de l'E.N.G.R.E.F., créée à Paris. L'Office national des forêts est mis sur pied, en même temps que les Directions départementales de l'agriculture. Les nouveaux organismes sont mieux adaptés aux tâches d'aménagement du territoire. Leurs missions spéciales se multiplient : développement des parcs nationaux et régionaux, reboisement économique du Massif central, délimitation claire des espaces agricoles et forestiers, remembrements pour regrouper les bois en forêts associatives, participation à l'organisation des nouveaux littoraux touristiques. Au fil du temps, à partir de la simple gestion régalienne des usages seigneuriaux, une administration du paysage s'est ainsi mise en place, les surfaces vaines se muant en cadre de vie.

Pour avoir finement saisi cette évolution, l'ouvrage consacré aux Eaux et Forêts du $12^{e}$ au $20^{e}$ siècle mérite une attention particulière. Dégageant une vue d'ensemble, il constitue aussi une référence essentielle pour les chercheurs, par l'abondance des sources et des données statistiques qu'il cite.

Jean-Marcel GoGER.

Françoise BAyARD, Le Monde des financiers au XnI ${ }^{e}$ siècle. Préf. de Pierre GOUBERT. Paris, Flammarion, $1988.14 \times 22,624$ p., index, bibliogr. ( Nouvelle bibliothèque scientifique $"$ ).

Voici un livre important qui contribue au même titre que ceux de Daniel Dessert (sur les financiers au temps de Louis XIV ou Fouquet) et de Joseph Bergin (sur la fortune de Richelieu) à démonter les ressorts les plus complexes et les plus secrets du « doux maniement des finances " (l'expression est de Cardin Le Bret dans son grand traité politique De la souveraineté du Roy, Paris, 1632).

Les 552 volumes du Conseil des finances pour la période 1593-1653 (série E) accompagnés des séries KK (les comptes de l'épargne) et $\mathrm{G}$ (administration financière) des Archives nationales permettent de suivre, au quotidien en quelque 
sorte, la gestion hasardeuse des revenus de l'État. Nous découvrons un roi sans gloire, incapable de chiffrer et de prévoir ses besoins comme le démontre amplement la pratique courante des "comptants » - un simple billet du souverain adressé au Trésorier de l'Épargne pour une dépense inattendue et qui ne réclame aucune justification - qui fait le désespoir des "bons » surintendants (comme Sully) et des historiens des finances... Progressivement, la monarchie se trouve prisonnière d'un groupe de quelques centaines de spécialistes appelés " traitants " ou «partisans » qui lui avancent les espèces sonnantes et trébuchantes nécessaires à sa politique de guerre et de prestige, ou tout simplement au fonctionnement quotidien des institutions.

De longs chapitres décrivent avec précision les mécanismes tortueux et obscurs - " tout ici n'est qu'apparence" (p. 16) - des dons d'avis, des fermes, des traités, et des prêts qui se greffent sur le système « normal » des impôts pour peu à peu le parasiter et le paralyser : les tailles sont mises en traités en 1643, l'État fait banqueroute au début de la Fronde.

S'il faut regretter pour la commodité de la lecture l'absence de tableaux qui auraient avantageusement remplacé de trop longues pages de chiffres et de pourcentages, on trouvera dans ce livre (qui fut une thèse) des développements neufs sur le " budget » de la monarchie, les extraordinaires et dévorants besoins nés de l'intervention indirecte (1630) puis directe (1635) de la France dans la guerre de Trente Ans : parce que « les nerfs des batailles sont les pécunes », c'est bien la guerre qui a modifié structurellement le système fiscal en aliénant définitivement l'État aux financiers pour cause de «resserrements » d'argent. En juin 1636 - l'année de Corbie et du Cid -, l'Épargne ne possède en caisse que $0,47 \%$ des besoins de la monarchie; en 1640 , on tente de boucler le budget de 1637 , en 1644 celui de 1641 ; en 1646 , le roi décide de percevoir la somme de 962850 livres sur la recette générale des finances de Poitiers de... 1651 .

Bon nombre d'idées reçues se trouvent ici ruinées : ainsi les recettes ordinaires - centrées essentiellement sur les différents modes de prélèvement de la taille ou du fouage (en Bretagne) - sont minoritaires dès 1605, c'est-à-dire que la formidable expansion des revenus de la monarchie - rappelons que les recettes en 1575 peuvent être évaluées à 15 millions de livres, en 1635 à 208 millions de livres! - s'explique surtout par l'accroissement vertigineux des recettes « souples " : les parties casuelles (les offices, officiellement héréditaires depuis 1604) et les deniers extraordinaires, ceux-là mêmes (rentes en particulier sur les villes, traités sur les impôts « indirects" - gabelle, aides, traites - puis " directs ", prêts) dont le fonctionnement est entièrement monopolisé par les financiers. Françoise Bayard démontre aussi que les dépenses les plus critiquées, celles de la cour, tendent à diminuer et ne dépassent jamais à partir de $163510 \%$ des dépenses de l'État. En ces temps où les intérêts privés entretiennent des relations si étroites avec les intérêts publics - l'étude récente de Joseph Bergin sur les affaires de Richelieu est sur ce point exemplaire - , nous découvrons au fil des chapitres l'ampleur des liaisons souvent dangereuses (et le plus souvent secrètes) que noue la "bonne société " du royaume - bourgeoisie, noblesse de robe ou d'épée, voire évêques et cardinaux - avec l'État dans la mesure où c'est elle qui avance aux financiers, par l'intermédiaire de prête-noms ou d'hommes de paille, 
une partie des sommes demandées par le roi. C'est ainsi que « l'État réussit ce tour de force inouil de faire payer volontairement les riches " (p. 452). Faut-il préciser que cette situation une fois instituée rend toute réforme de structure impossible, de Richelieu (pages éclairantes dans son Testament politique) à Calonne...

C'est la première fois aussi qu'est dressé avec autant de précision - grâce aux archives notariales qui une fois encore démontrent leur fécondité - le portrait collectif de ces 5000 hommes que les contemporains appellent « sangsues du peuple " ou " laquais financiers ", victimes régulières de " purges " que constituent les Chambres de justice qui innocentent l'État de toute responsabilité dans la "mauvaise " gestion de ses finances en punissant des boucs émissaires et dissimulent le plus souvent des règlements de compte politique et des changements de «lobbys" (Richelieu contre La Vieuville en 1624, Colbert contre Fouquet en 1661 ...). Ces financiers - certains comme Sébastien Zamet au temps de Henri IV qui l'appelle familièrement « Bastien ", ou Montauron, à qui Corneille dédicace Cinna ou la Clémence d'Auguste en 1639, sont particulièrement célèbres - appartiennent en majorité au monde des officiers de justice et de finance (de moins en moins la justice, de plus en plus les finances). Ils sont le plus souvent parisiens (ou lyonnais comme la dynastie des Mascranny-Lumague) et s'ils s'intéressent relativement peu à l'économie, sauf en période de paix, ils sont pleinement intégrés aux rouages de l'État : fidèles au roi - leur cabinet de travail est souvent orné de son portrait - , ils observent une grande réserve lors des événements - la Ligue, la Fronde - qui mettent en péril la monarchie. Surtout, ils s'identifient parfaitement par leur mode de vie ostentatoire à la société aristocratique ; mais le crédit public et le crédit privé ne sont-ils pas, ainsi que le clamera en vain lors d'un procès scandaleusement truqué Fouquet, indissolublement liés? Par leur comportement, leurs achats de livres et de tableaux, ils participent aussi pleinement aux grandes mutations intellectuelles et idéologiques du temps comme la Réforme catholique dont beaucoup sont d'ardents et de dévots militants.

Avec des travaux comme celui-ci, et en attendant les résultats des recherches voisines d'Alain Guéry qui s'attache à reconstituer l'ensemble du budget de la monarchie sur trois siècles, c'est un nouveau territoire de recherche qui vient d'être brillamment investi.

Joël CORNETTE.

LAVOISIER, De la richesse territoriale du Royaume de France. Texte et documents présentés par Jean-Claude PERrot. Paris, Ed. du C.T.H.S., 1988. $12 \times 18,5$, 271 p. (« C.T.H.S. Format $3 »)$.

Dans la collection des rééditions au format de poche qu'a créée depuis peu le Comité des travaux historiques et scientifiques, voici les fragments d'une grande 
œuvre. Le texte même de Lavoisier tient en soixante pages, le reste étant occupé par une savante introduction de Jean-Claude Perrot qui apporte également, en annexes, commentaires et documents pour éclairer la lecture. Le livre publié en 1791 se présente comme les " Résultats extraits... " d'un ouvrage à venir. L'Assemblée constituante qui, dans l'hiver 1790-1791, se préoccupait d'établir la contribution foncière, avait décidé de publier les éléments de calcul que Lavoisier avait soumis au Comité des impositions. II ne s'agissait pas pour elle de les avaliser mais d'encourager et d'honorer une recherche, en versant cette pièce au débat. Ainsi fiscalité et statistiques sont-elles associées dans une même démarche, au risque d'être confondues dans une même réprobation. Le temps ne devait pas être laissé à Lavoisier d'élargir son propos; il nous reste au moins ces pages à méditer.

L'enjeu de ce texte, où se rencontrent l'Assemblée et le savant, est d'établir le revenu monétaire annuel, sur lequel doit reposer la contribution foncière. Ce revenu net est une résultante du produit brut; l'objectif est donc d'évaluer celui-ci, c'est-à-dire de comptabiliser tous les types de récoltes. A partir de cette base, il sera possible, en évitant les doubles emplois, de passer du produit physique à sa valeur monétaire puis, en défalquant les dépenses, au revenu. Comment y arriver? La voie directe serait de saisir les volumes produits en croisant surfaces cultivées et rendements. Mais ces données sont très difficiles à cerner et fluctuantes. Lavoisier en est conscient. Il pense contourner l'obstacle en visant la consommation, posée comme équivalente, en année commune, à la production. Et pour atteindre la consommation, il y a un élément connu, accessible, la population. La méthode, en somme, est de trianguler le territoire de l'économie. La population forme la base et permet de calculer les deux autres côtés, réputés égaux. Au commencement était la démographie.

La méthode de Lavoisier s'appuie sur l'information la plus solide, au moins le pense-t-il, réunie par Moheau et d'autres. Mais, pour son projet, il a besoin d'un instrument plus raffiné que des dénombrements. Car il y $a$, estime-t-il, des structures diversifiées de consommation, par sexe, âge, habitat et profession. Au lieu de prendre, selon un « topos » en vogue, la ration du soldat à titre d'unité de compte, il s'astreint donc à chercher dans les données déjà établies les moyens de cette distinction. Autre voie d'accès, celle des budgets familiaux. Inaugurant ici l'approche monographique, qui sera systématisée, au siècle suivant, par Frédéric Le Play, Lavoisier montre tout le profit que l'on a à établir les types de consommation de ces groupes primaires, selon leur place dans l'échelle sociale. Mais contrairement à Le Play et à ses continuateurs, Lavoisier établit un va-et-vient entre comptabilité nationale et études de cas, celles-ci validant celle-là. La dernière piste est plus banale mais pas moins prometteuse : il s'agit d'examiner le poids de Paris dans l'agrégat final. Ici les sources affluent; le fermier général sait la bonne fiabilité des comptes d'entrée des denrées dans la ville. Il dresse ainsi un tableau détaillé des consommations où apparaît l'écart parisien à la moyenne nationale.

On passera sur d'autres éléments, que Lavoisier tire de son expérience de propriétaire terrien, pour examiner les résultats et les enseignements de son entreprise statistique. Partons du chiffre "politique ", c'est-à-dire du revenu 
imposable. Lavoisier établit celui-ci à 1 milliard 200 millions de livres, qu'il réduit à 1 milliard, en anticipant une baisse du prix des grains. C'était mettre le rendement de l'impôt à moins de 200 millions, puisqu'il était entendu que le prélèvement serait de l'ordre du sixième. L'Assemblèe ne pouvait accepter un résultat aussi faible, compte tenu des besoins de l'État. Elle décréta donc une contribution de 300 millions, faisant comme si le revenu foncier s'établissait à 1 milliard 800 millions. Relativité de la science économique... Mais l'analyse interne de la comptabilité agricole dans la version de Lavoisier montrait qu'elle souffrait, comme le pressentaient les contemporains, d'une sous-estimation sérieuse. Nous en savons la cause : Lavoisier évaluait à 25 millions d'habitants la population du Royaume, suivant en cela la majorité des observateurs, alors qu'elle atteignait probablement 27 millions et demi de personnes en cette année 1790 . Dès lors, sans même interroger d'autres paramètres, on est conduit à réviser en hausse - mais pas autant que l'Assemblée a cru devoir le faire! - les calculs de Lavoisier.

Plus que les résultats importent la méthode et le programme. L'ambition, en effet, est immense. Elle est d'élaborer, à terme, une véritable comptabilité nationale suivant au plus près, chaque année, l'évolution du produit. Lavoisier appelle à la réalisation de ce qui serait un " thermomètre de la prospérité publique". Comment parvenir à créer cet instrument d'une gestion éclairée ? Ici la politique donne la main à la science. C'est parce que le Royaume s'est doté d'une nouvelle organisation des pouvoirs, ramifiée et cohérente, que cette collecte devient possible : «avec une correspondance patriotique de cette espèce, il n'est point de renseignements que l'on ne puisse obtenir, point de travaux qu'on ne puisse entreprendre. » Ce faisant, le grand chimiste est convaincu d'aider la science de l'économie politique à sortir de l'enfance, de l'âge métaphysique. Constituer ces données, réunir les matériaux de l'édifice, c'est passer, enfin, de la théorie des philosophes à la pratique de l'homme d'État. Ce propos sévère est aggravé, si l'on peut dire, quelques pages plus loin. Écoutons Lavoisier : " Un travail de cette nature contiendrait en un petit nombre de pages toute la science de l'économie politique, ou plutôt cette science cesserait d'en être une; car les résultats en seraient si clairs, si palpables, les différentes questions qu'on pourrait faire seraient si faciles à résoudre, qu'il ne pourrait plus y avoir de diversité d'opinion. " S'agit-il pour Lavoisier de laisser entendre que cette science accéderait au statut de technique, en quittant l'incertitude qui est au cceur de son objet social? Est-ce la fin de l'économie politique, dans la transparence de l'administration des choses? Le commentaire de Jean-Claude Perrot, si précieux pour le reste, est bien discret sur cette ouverture. Est-ce pour respecter le mystère d'une œuvre inachevée?

Denis WORONOFF. 
Hervé Dumez, L'Économiste, la science et le pouvoir. Le cas Walras. Préf. de Henri GurTION. Paris, P.U.F., 1985. $15 \times 21,5,272$ p., bibliogr., index ("Sociologies").

Que le lecteur ne s'attende pas, dans cet ouvrage, à trouver une biographie traditionnelle de l'économiste Léon Walras. Non seulement le récit linéaire de son existence ne s'y trouve pas mais, en plus, l'auteur ne s'est intéressé ni aux caractéristiques intellectuelles de l'économiste ou de son milieu, ni aux conditions sociales de production de sa doctrine et encore moins au contenu même de son cuvre. Sa démarche est autre et s'inspire de la méthode présentée par R. Boudon ${ }^{13}$ et $\mathrm{F}$. Bourricaud ${ }^{14}$, qui consiste à concevoir l'intellectuel comme un producteur opérant sur un marché. Comment et dans quel milieu s'est diffusée la théorie walrasienne? Comment Walras, en tant qu'émetteur d'une doctrine révolutionnaire, a-t-il pu s'intégrer à la corporation reconnue des économistes?

Dans une première partie, $H$. Dumez procède à une véritable « analyse de marché " (il est lui-même chercheur au Centre de recherches en gestion de l'École polytechnique). Il passe en revue la demande et l'offre d'idées économiques en France vers $1860-1880$ et la position des idées walrasiennes sur œe marché. A une demande diffuse, multiple mais mal formulée, en particulier par l'État aux prises avec la question sociale et les exigences d'un interventionnisme croissant, doit répondre une offre mal adaptée. Deux écoles théoriques dominent en France au début de la $\mathrm{II}^{\mathrm{e}}$ République : l'école libérale et l'école historique. La première doit assumer l'héritage de Smith, Say et Bastiat, constitué autour du dogme de la concurrence, loi naturelle qui dépasse l'économie politique et garantit son caractère scientifique. Il en résulte, d'une part, l'absence de prise en considération des apports de Cournot qui, le premier, mathématise l'économie par l'étude des lois du monopole et, d'autre part, un blocage théorique presque total puisque les grands ancêtres ont " achevé » la science. L'essor de la deuxième résulte du choix effectué par les professeurs de droit mués en professeurs d'économie politique (surtout après 1877, quand les Républicains font de la discipline une matière d'examen dans le cursus juridique) qui trouvent avec l'école historique une méthode qui n'est plus théorique et qui reste proche de leur savoir traditionnel. Il en résulte une inadéquation entre offre et demande puisque les blocages de celle-là l'empêchent de répondre aux exigences variées, et tout particulièrement techniques, de celle-ci.

Le dernier chapitre de la deuxième partie étudie l'adaptation des idées walrasiennes aux exigences théoriques et techniques du temps. L'auteur souligne la rupture qu'elles constituent, facteur essentiel du rejet de Walras par les milieux scientifiques. Elle est due surtout à la formalisation mathématique puisque Walras, tout au contraire de Marshall, adopte une stratégie "dure " avec la mise au premier plan de l'appareil mathématique. Si les économistes littéraires n'acceptent pas cette modélisation, celle-ci répond parfaitement à la demande technique

13. Effets pervers et ordre social, Paris, P.U.F., 1977.

14. Le Bricolage idéologique, Paris, P.U.F., 1980. 
des ingénieurs (gestion et calcul économique) dont l'entrée dans le milieu scientifique de l'économie est considérée par $\mathrm{H}$. Dumez comme un événement majeur pour la discipline surtout en France.

C'est bien l'un des points centraux de cet ouvrage que de montrer l'importance pour le succès des idées walrasiennes de ce que Schumpeter appelle « les résultats de détail dans des domaines fort divers " (p. 100). L'auteur illustre fort bien cet aspect avec la longue analyse de l'article de 1875, "L'État et les chemins de fer ", qu'il considère comme l'un des chefs-d'œuvre du maître de Lausanne (p. 100113).

Dans la deuxième partie, l'auteur essaie de voir comment le processus d'émergence de l'économie mathématique est marqué par les aventures personnelles de son inventeur et par sa stratégie. Dans un premier temps, il dresse un portrait de Walras en 1870, à trente-six ans, date à laquelle il se présente au concours ouvert pour pourvoir la chaire d'économie politique à Lausanne. Deux traits apparaissent essentiels. D'une part, l'influence intellectuelle de son père, le philosophe Auguste Walras. Celui-ci lègue à son fils une théorie de la valeur hétérodoxe qui implique le calcul de la rareté d'un objet échangeable, exigence qui conduit à la mathématisation walrasienne. De même, l'économie appliquée et les préoccupations sociales (en particulier, la fiscalité) du fils résultent de l'influence du père. D'autre part, $H$. Dumez présente un portrait de l'économiste en « jeune recalé » (p. 127). Jeune recalé à Polytechnique, incapable de finir l'Ecole des mines entreprise comme élève externe, Walras a une revanche à prendre. Il est certain que celle-ci réside dans la réussite de la mathématisation d'un domaine informe et son complexe vis-à-vis des mathématiques - dans lesquelles il n'excellait d'ailleurs pas, comme il est souligné à de nombreuses reprises - explique en partie son manque de concession dans la présentation trop technique de ses théories pour une science peu habituée à la formalisation. Ces difficultés initiales de carrière induisent des complications aussi matérielles que professionnelles que l'auteur retrace rapidement mais avec une certaine réussite (p. 130-139). Les deux chapitres qui font suite permettent de mettre en parallèle deux tentatives d'insertion dans un milieu universitaire dans des cadres très différents : le canton de Vaud et la France. Le passage sur Walras à Lausanne vise, en effet, à démontrer que la grande université suisse ne fut pas un simple lieu d'accueil mais un élément important dans la réussite intellectuelle du père de l'équilibre général. L'auteur met en lumière la politique universitaire suivie par le Conseil d'État vaudois qui repose sur la notion de contrat passé avec le savant recruté. Celui-ci est nommé sur la base d'un programme de recherche (longuement présenté dans l'ouvrage) qu'il devra poursuivre et enseigner, moyennant une sécurité professionnelle qui donne liberté et longueur de temps. Le conseiller d'État Louis Ruchonnet, qui contribua beaucoup à la venue en Suisse de Walras, est le personnage central de ce chapitre, présenté comme un modèle d'homme politique dans ses rapports avec la science et les scientifiques. Le deuxième volet, français, contraste beaucoup. Walras, soucieux de revanche intellectuelle et universitaire, désirait occuper une chaire en France dans un établissement prestigieux. H. Dumez décrit en détail ses multiples échecs en particulier au Collège de France et à l'École pratique des hautes études. L'énergie déployée et la futilité des causes de ses nombreux échecs 
apparaissent avec évidence; la notion de projet scientifique, si essentiel à Lausanne, semble absente des grandes décisions universitaires prises en France. Mais l'auteur a quelques difficultés pour développer l'analyse au-delà de la simple comparaison descriptive. Il est, par contre, beaucoup plus convaincant quand il oppose « les deux ministres » (chap. 8), Ruchonnet et Jules Ferry. Celui-ci est un ami personnel de longue date, sincèrement intéressé aux idées de Walras et désireux de l'aider pour son retour en France. II manque cependant constamment de temps pour lire les cuvres de l'économiste autant que pour lui fournir des appuis significatifs. De plus, sa longue action politique est une succession de préoccupations urgentes (en particulier, la campagne anticléricale) qui exclut toute politique à long terme dans un domaine aussi mineur pour lui que l'enseignement de l'économie. Alors qu'à Lausanne le pouvoir semble au service de la science, celle-ci en France si elle est neuve, non reconnue institutionnellement et qui plus est portée par un individu sans diplôme, n'apparaît alors d'aucune utilité. Walras est obligé de tenter de prouver à Ferry que sa nomination en France, par le " renom historique » qu'elle aura, peut constituer un enjeu de carrière. L'enjeu de la science se trouve, lui, bien absent.

Le dernier chapitre étudie encore la stratégie de Walras, face au milieu scientifique. Comment " vendre " sa doctrine quand on est en possession d'une théorie révolutionnaire? Tous les aspects du problème sont évoqués avec les difficultés initiales d'édition (résolues grâce à l'appui de Ruchonnet) jusqu'au rayonnement international et les concurrences inévitables (en particulier avec Jevons), en passant par la nécessaire communication à l'Académie des sciences morales et politiques, les discussions critiques dans les revues françaises, la recherche de cautions mathématiques indiscutables comme celle de Poincaré à qui Walras envoie en 1901 la quatrième édition des Éléments d'économie pure et, enfin, l'échec significatif du premier disciple français, Aupetit, au concours de l'agrégation. Cet échec affecta gravement Walras qui y vit un insuccès de plus dans la pénétration en France de ses idées. Bref, $\mathrm{H}$. Dumez décrit avec détail le parcours du combattant que doit subir un économiste en mal de renommée. Loin de l'image idyllique du savant au génie précoce et exceptionnel, détaché d'une réussite qui s'impose à lui, Walras apparait au contraire comme écrasé par l'image des grands scientifiques comme Kepler ou Newton, dépourvu de dons supérieurs dans son domaine de prédilection, les mathématiques, et obligé de promouvoir une carrière qui, jusqu'à la fin, resta bien en dessous de ses ambitions.

Cette lecture appelle trois remarques. En premier lieu, ce travail présente un intérêt évident sur le plan factuel sur un sujet en fin de compte peu étudié. En second lieu, la démarche de l'auteur mérite attention. Ni biographie ni étude sociologique d'un milieu, ce travail est centré sur la problématique de l'insertion de l'individu, dont l'originalité ici est d'être un savant marginal, dans un groupe social spécifique bien qu'aux contours flous, celui des universitaires, décideurs ou hommes politiques en rapport avec l'économie politique. Un regret et une difficulté apparaissent alors. D'une part, les spécificités de l'expérience walrasienne et la généralité de ses enseignements apparaîtraient mieux si des comparaisons plus fréquentes étaient faites avec d'autres scientifiques, au lieu de trop rares allusions à Einstein, Marshall ou Jevons. D'autre part, les mécanismes sociaux 
doivent être perçus en tant que tels, sans être réduits à l'action de tel ou tel individu. Or cette rigueur, difficile à atteindre, est parfois absente, ce qui donne l'impression de retomber dans les facteurs explicatifs traditionnels comme l'influence du père ou des amis efficaces (comme Ruchonnet) ou non (comme Ferry). De même, la notion de marché avec ses nécessaires régularités sociales disparaît dans la deuxième partie au profit de l'action favorable ou hostile de tel ou tel individu. Bref, l'individu n'est plus posé face au groupe social mais face à d'autres individus, au détriment de la pertinence sociologique. En troisième lieu, on peut s'étonner de l'absence de la dimension épistémologique. Il ne s'agit, certes pas, du propos de l'auteur puisque son ouvrage est d'abord sociologique. On peut se demander tout de même si, dans cette perspective même, cette dimension n'aurait pas été nécessaire. Ainsi l'apport de Walras est trop réduit à la seule mathématisation, ce qui limite les oppositions à Walras au seul clivage formalisation mathématique/approche littéraire. Clivage évident, il laisse cependant dans l'ombre d'autres spécificités walrasiennes, en particulier l'idée d'équilibre général, qui ont dû contribuer à son positionnement propre sur le marché de l'économie politique.

Ces remarques ne retirent rien à l'intérêt de ce livre souvent novateur. Elles sont, au contraire, les conséquences inévitables d'une approche nouvelle et nécessaire en histoire de la pensée économique qui mériterait d'être élargie à d'autres auteurs et à d'autres époques.

Jean-Yves GrenIER.

Le Capitalisme français : $19^{e}-20^{e}$ siècle, blocages et dynamismes d'une croissance. Dir. Patrick Fridenson et André Straus. Paris, Fayard, 1987. $16 \times 24$, $427 \mathrm{p}$.

Cet ouvrage collectif auquel ont participé vingt-deux universitaires, économistes et historiens constitue un événement important si l'on considère à la fois la production intellectuelle dans ces domaines de la recherche, et l'évolution de la vision de l'économie, de son passé et de son présent, par les Français. Ainsi que le mettent en évidence Patrick Fridenson et André Straus dans l'avant-propos du recueil, « les années récentes ont vu un considérable retournement de l'opinion publique française concernant l'économie. En peu de temps, tout a changé. Les contraintes internationales ont cessé d'être niées, l'État-Providence a été remis en question; l'ouverture sur le grand large, la concurrence, l'entreprise, le patronat, suscitent désormais des réactions très positives ». Les conséquences de cette révision fondamentale des attitudes de nos concitoyens à l'égard des réalités économiques sont aussi importantes que l'est l'apparition d'une nouvelle histoire économique «... à la française, solide autant que pluraliste », ainsi que le soulignent les auteurs. La démarche de ces historiens se veut "révisionniste " quant au cas français, démarche qu'ils partagent avec Jean Bouvier autour duquel les auteurs du livre ont tous travaillé.

Révisionniste, l'approche l'est sans conteste, libérée du double blocage et du 
carcan des idées préconçues sur l'économie française, d'une part - "son déclin inéluctable et [son] malthusianisme ravageur " - du marxisme, d'autre part. C'est là l'occasion de saluer un courage intellectuel indéniablement représenté par Jean Bouvier, et une réappréciation des données de base. Aussi la méthodologie estelle l'enjeu essentiel de ces recherches, qui doivent permettre un bon usage des contradictions.

La croissance, comme l'exprime Jean Bouvier dans l'introduction de l'ouvrage, significativement intitulée "Libres propos autour d'une démarche révisionniste ", ne s'oppose pas aux blocages, il y a croissance malgré les blocages, grâce aux blocages. L'important est là : aucune détermination n'existe $a$ priori, matérielle, sociale ou culturelle qui expliquerait que notre économie et notre société aient été durablement bloquées depuis le XVIII ${ }^{\circ}$ siècle. Le capitalisme français n'accuse ainsi aucun retard (initial, ni même par la suite) par rapport aux modèles successifs anglais, allemand, voire japonais.

Il en est ainsi des présupposés comme celui du sacrifice de l'efficacité économique à la stabilité des campagnes, par la consolidation des petites exploitations rurales du fait des Biens nationaux et du Code civil. Les structures agraires n'ont jamais connu, ni au $\mathrm{XIX}^{e}$ ni au $\mathrm{XX}^{e}$ siècle, de "blocage absolu", mais une évolution lente à la mesure de conditions objectives (climats et sols, démographie, structures sociales et foncières bien antérieures à 1789). J. Bouvier élude également une approche trop systémique des rapports entre l'économie et la démographie : pas de monocausalité mais une croissance économique honorable sans effet mécanique de la population sur la demande. Et cette croissance, si elle n'est pas condition suffisante, est condition nécessaire des progrès sociaux.

L'ouvrage se lit à la lumière de cette attitude révisionniste, " ambiguë » mais indispensable, qui élève à un niveau apprécié la recherche des rationalités. C'est d'abord l'étude des rapports entre l'Etat et l'économie. Un État qui, au XX⿳亠丷厂 siècle, a d'autant plus tendance à intervenir et à empiéter sur les décisions privées et les mécanismes du marché, quand celles-ci ou ceux-ci ne fonctionnent pas, fonctionnent mal ou vont à l'encontre de l'intérêt général. Pierre Goubert ne disait-il pas déjà à propos du $x^{\prime}{ }^{e}$ siècle que " ...l'Etat n'intervient pas quand les affaires vont toutes seules et, par corollaire, [que] le colbertisme est le signe même de la contraction économique $"$ ?

Pierre Saly présente un Poincaré keynésien avant terme et sans le savoir, relançant la demande globale, à la fois par une revalorisation du salaire réel obtenue par la stabilisation de la monnaie - et par les effets des investissements publics; la prospérité française de la fin des années vingt aurait ainsi été permise par une "stimulation discrète de la demande globale » et non par une orthodoxie financière et budgétaire.

L'administration du Trésor est décrite par Michel Margairaz en termes d'autorité au-delà des interventions techniques et d'une mutation intellectuelle fondamentale entre 1930 et 1950 . L'influence d'un Jacques Rueff sur la politique économique et financière entre 1936 et 1938 est indéniable; la direction du Trésor freine l'engagement de l'État en faveur de la croissance. A partir de 1948, le risque socio-politique dissipé permet au Trésor de décider du risque financier. Henry Rousso démontre qu'au-delà du dirigisme théorique élaboré par Vichy, le régime 
ne peut modifier les structures économiques du pays, du fait des archaïsmes mais bien plutôt des contraintes de l'Occupation et de la situation économique désastreuse. S'il y a modernité, c'est dans l'ébauche de rationalisation des décisions, en continuité avec les projets d'avant-guerre et ceux de 1944-1945.

L'après-guerre voit le ministère de l'Industrie tenter d'imposer aux entreprises sidérurgiques, par l'intermédiaire du Plan Monnet, modernisation et restructuration. Les réticences de ces entreprises aboutissent selon Philippe Mioche à une modernisation « ni contre l'État ni par l'État » mais « avec lui ». Qu'il aide, qu'il incite, qu'il permette, l'État est devenu l'acteur majeur.

La deuxième partie de l'ouvrage traite des atouts de l'industrialisation. A partir de l'étude d'entreprises spécifiques, les Forges et Acieries du Nord-Est de 1917 à 1948 et Saint-Gobain de 1945 à 1969, Odette Hardy et Jean-Pierre Daviot remettent une fois de plus en cause la thèse du malthusianisme du patronat industriel, souvent capable d'une incontestable adaptabilité. La modernisation dans les entreprises publiques s'effectua d'après P. Fridenson " par le haut " entre 1944 et 1986, dans les domaines de la technologie, de la gestion, des aspects sociaux. Modernisation limitée, fonction de la diversité des entreprises, et de leur insertion dans les marchés mondiaux, mais rôle actif dans la modernisation des relations sociales, dans la priorité au marché - pour les banques - et dans la constitution de groupes de taille internationale : le moteur semble bien l'avoir emporté sur le frein.

Dans le domaine bancaire, la contribution d'Alain Plessis ne permet plus de soutenir, ainsi que le fit Rondo Cameron dans les années soixante, la thèse d'une " corrélation étroite " entre le développement tardif de nos structures bancaires et la marche également lente de l'industrialisation du pays. Il n'y a pas de « retard français » du système bancaire par rapport à l'Angleterre sous le Second Empire. Le système anglais est plus concentré, avec ses 1628 bureaux dépendant de 365 banques; en France, on compte plus de 3000 guichets, pour la plupart dépendant de banquiers locaux, mais dont la valeur des effets de commerce fait plus que de tripler entre 1845 et 1869 : le crédit à l'économie n'a pas été rationné. Pas plus qu'il ne l'a été dans l'agriculture, par le biais des banques mutualistes, avec l'aide de l'État (André Gueslin); le crédit aux petites et moyennes entreprises, qui font le tissu industriel de la France de l'immédiat avant-guerre, semble bien avoir été plus limité (Michel Lescure). Enfin, la " nationalisation du crédit ", c'est-à-dire la direction du crédit confiée à la Banque de France nationalisée en 1945, n'a pas eu, d'après Claire Andrieu, un effet négatif sur la croissance, au contraire; la Banque de France, demeurée une entreprise, même nationalisée, a contenu le crédit, mais aussi participé à l'action de reconstruction et de modernisation par sa politique de taux qui s'apparenta à un néo-libéralisme de facto entre 1945 et 1950 .

La croissance économique française s'inscrit dans un contexte de contraintes extérieures de plus en plus fortes. Contraintes monétaires d'abord, qui s'expriment en termes de rapports de force. Jean-Claude Deiber évoque la « coopération obligée » entre la Banque de France et la Banque d'Angleterre entre 1926 et 1931, à la suite de la dégradation des relations franco-britanniques du fait des réparations allemandes, et qui révèle des échanges d'information et un rôle monétaire 
grandissant de la France. Cette coopération permet à la France de tenir une place de premier rang dans la finance internationale : l'exportation de capitaux réalise la condition principale de la force du système économique français, pendant que se déroule - entre 1913 et 1930 - la plus forte croissance industrielle d'Europe. Ainsi, l'intégration du pays dans les circuits financiers internationaux ne s'est pas faite au détriment de la croissance interne.

Cette coopération monétaire est rendue obligatoire à partir de 1947 pour les pays européens, du fait de la pression américaine et l'on peut alors avec Robert Frank parler de «contraintes monétaires " dont le Plan de modernisation doit permettre de s'affranchir, dans un effort national qui n'intègre que très mal la nouvelle dimension européenne, à l'exception des « minoritaires courageux que sont ces décideurs français, Schuman, Monnet, Bonnet et la Société des Européens ", comme l'explique Annie Lacroix-Riz.

Le lien entre l'économie internationale, la croissance économique et la colonisation est évoqué par Jacques Marseille. Le mouvement de colonisation du dernier quart du $\mathrm{xrx}^{\mathrm{e}}$ siècle n'est pas le produit d'un stade du capitalisme : l'exportation de capitaux s'explique par une succession de régulations, « concurrentielles » et dirigées vers des débouchés externes, "monopolistes » et affirmant des débouchés internes. Pendant la régulation concurrentielle, les industries du textile, de la métallurgie négligent les investissements, tournées qu'elles sont vers les colonies; la phase de régulation interne du capital implique une conversion effectuée et réussie à partir de 1950 pour la France, vers des industries à plus forte valeur ajoutée.

L'analyse porte in fine sur les structures et les mentalités, présentées alternativement comme des freins ou des dynamiques. Les viticulteurs méridionaux étudiés par Geneviève Gavignaud présentent des capacités de résistance fondées sur la famille, la coopérative, l'État. Ce sont d'autres valeurs, telles que la culture de l'entreprise, qui caractérisent les pouvoirs des gens de Pont-à-Mousson : ni force, ni faiblesse, elles sont tantôt l'une et tantôt l'autre.

Au terme de la lecture de cet ouvrage, divers par ses sujets comme par ses démarches d'analyse et d'exposition, on pourra penser qu'il s'agit bien là d'une " revalorisation de l'histoire économique dans notre pays ». Par ses questions, par ses désaccords quelquefois, par ce mouvement réussi d'une mise en perspective permanente et d'un singulier retour au concret, il permet la révision de la collection et de l'interprétation des faits. Il y parvient en refusant, ainsi que le propose J. Bouvier, « ... toute mono-discipline, toute mono-théorie, tout impérialisme intellectuel ». Il met brillamment en évidence les moteurs de la croissance française, contribue à définir avec davantage de précision une identité française qu'eût appréciée Fernand Braudel, pour qui « ... le travail de l'historien c'est de complexifier le réel, non de le simplifier ".

Tristan LECOQ. 
Christian Topalov, Le Logement en France : histoire d'une marchandise impossible. Paris, Presses de la Fondation nationale des sciences politiques, 1987. $14 \times 22,456 \mathrm{p}$.

Avec un ouvrage dont le sous-titre peut paraitre, de prime abord, quelque peu énigmatique, C. Topalov entreprend de nous narrer, dans la longue duree, l'histoire des amours difficiles de la pierre et du capitalisme. Si le logement est décidément une marchandise impossible, c'est qu'il ne s'agit pas d'un bien comme les autres. Sa production suppose la mobilisation d'un matériau rare, non reproductible à l'infini et retenu par des propriétaires privés : le sol ; sa diffusion se heurte à un goulet d'étranglement : le pouvoir d'achat des consommateurs et, plus précisément, des couches populaires, seules aptes, ici comme ailleurs, à sécréter un vaste marché. Pour ces deux raisons, la construction capitaliste est condamnée à se porter vers les segments supérieurs du marché, donc à limiter sa clientèle, ou bien à intégrer à un endroit ou à un autre du processus de production des agents non capitalistes, bref à se nier elle-même. L'histoire du logement apparaît finalement comme le récit des tentatives vaines pour surmonter cette contradiction, pour guérir de cette véritable maladie de la pierre.

Pour inaugurer son parcours, l'auteur suit la lente mais inexorable transformation de l'habitat en marchandise. Il ne remonte pas jusqu'à l'Antiquité et encore moins jusqu'au Néolithique, mais s'enfonce dans le passé mythique du haut Moyen Age, au temps où la propriété ne circule pas librement, enserrée dans les mailles du féodalisme. Progressivement, par des voies multiples, l'habitation se dégage de ses entraves, elle se transmet bientôt librement aux descendants ou à d'éventuels acheteurs. Elle devient alors une marchandise avant que l'expropriation de ses occupants au $\mathrm{XIX}^{e}$ siècle ne la transforme en capital aux mains d'une classe de rentiers.

On accompagne aisément l'auteur dans sa démonstration. C'est tout juste si l'on peut s'interroger sur l'acte de naissance du logement-marchandise. N'y avaitil pas de transmission de la propriété urbaine dans la Rome antique ? Le marché des immeubles n'est-il pas assujetti à la croissance des villes, à la circulation de la monnaie, au crédit? Le logement devient-il ou redevient-il une marchandise ? Quoi qu'il en soit, un tel affranchissement du logement se réalise au prix d'un éclatement : les habitations se couvrent de rentes et les détenteurs de ces créances s'arrogent du même coup une fraction du titre de propriété. Il est vrai que la Révolution élimine ces redevances, aidée puissamment, il faut le dire, par la dépréciation de l'assignat.

L'évolution ultérieure n'est pas aussi favorable aux occupants. Ils deviennent locataires de leur maison et ce processus achève de mettre en place les conditions d'un système de production capitaliste du logement. C. Topalov se place alors délibérément à deux moments historiques privilégiés pour décrire les tentatives de développement de la production immobilière sur une base capitaliste et leur faillite. Une crise clôt chaque cycle et génère à terme, à chaque fois, un nouveau système de production.

La première phase correspond à la période 1860-1914 et coïncide avec ce que 
l'auteur appelle l'âge d'or du rentier. Pendant ce demi-siècle, l'investissement est assuré soit par des propriétaires patrimoniaux, soit par des promoteurs indépendants de la propriété foncière. Ce dualisme, primordial, en recouvre bien d'autres : géographiques, physiques, sociaux. Les premiers se spécialisent, en effet, dans le logement ouvrier, bon marché, peu élevé. Les seconds développent des immeubles de rapport, de haut de gamme, de type hausmannien.

Si le système entre en crise dès 1880 , c'est par l'extension du marché foncier, l'abstention croissante des rentiers et l'orientation progressive vers les segments supérieurs du marché. De là résultent deux effets pervers : la saturation du marché cher, la pénurie du logement bon marché, et cette inadéquation entre l'offre et la demande est responsable de la crise qui sévit pendant le demi-siècle suivant, bien plus qu'un blocage des loyers abusivement invoqué.

La seconde phase dure de 1950 à 1980. Elle s'identifie au succès de la promotion immobilière. Après une longue période de marasme, la construction reprend vigueur. Individuellement ou groupés en sociétés, les promoteurs mobilisent des capitaux, des terrains, des matériaux, pour produire et surtout pour vendre des appartements. Le moteur, effectivement, n'est plus la recherche d'un placement pour un rentier, mais l'effort des particuliers, pour accéder à la propriété d'occupation. Paradoxe : les habitants, expulsés pendant la Révolution industrielle redeviennent propriétaires sous la houlette du capitalisme. L'auteur décrit minutieusement le fonctionnement et la conjoncture de ce mode de production et montre qu'il impose sa norme aux autres formes de lotissement jusqu'à la crise de 1974.

L'effondrement de la production est concomitant avec l'avènement d'un nouveau système : la commande sur catalogue généralement à l'initiative des particuliers. De plus en plus dépendants du capital financier, exclus de ce type de construction, handicapés à nouveau par l'étroitesse du marché, les promoteurs perdent du terrain et la production se sépare du mode de production capitaliste, comme un siècle plus tôt. L'auteur y voit la preuve d'une impossibilité structurelle pour le capitalisme de s'imposer dans un secteur de production essentiel, puisqu'il commande pratiquement tous les autres. La consommation de masse du logement représenterait bien une utopie.

Ce modèle cohérent et séduisant a le mérite de rendre compte sur plus d'un siècle des transformations subies par le secteur immobilier en France et d'en expliquer la conjoncture. Comme tous les modèles, il comporte certaines limites que l'auteur perçoit d'ailleurs fort bien. De fait, il me semble que l'on peut difficilement échapper à deux interrogations majeures. $1^{\circ} \mathrm{La}$ construction immobilière représente-t-elle réellement un secteur de production autonome? Les grandes phases que l'on distingue sont-elles uniquement le produit de contradictions internes ou bien sont-elles étroitement liées à la conjoncture générale ? 1880 ou 1965 ne sont pas des dates innocentes non plus que 1974 d'ailleurs. $2^{\circ}$ En accordant d'emblée la priorité à l'offre comme vecteur de la production, ne sacrifie-t-on pas les autres agents économiques qui développent leur propre stratégie, en fonction de leur épargne disponible, de leur propension à consommer... J. Capdevielle a montré que l'acquisition d'un patrimoine représente largement un investissement métaphysique, la réponse à une angoisse 
existentielle ${ }^{15}$ et il en fait le fondement de la classe moyenne. Dans ce contexte, que peut bien impliquer une statistique qui révèle que l'accès à la propriété est resté constant au-delà des soubresauts des mises en chantier, pendant près d'une décennie ${ }^{16}$ ?

Pourtant il est certain qu'en choisissant délibérément un angle d'approche privilégié, en attribuant aux formes de production le rôle moteur, C. Topalov propose une interprétation tout à fait judicieuse et convaincante des destinées du bâtiment. Après d'autres auteurs ${ }^{17}$, il apporte sa pierre à l'édification d'une histoire du logement en France.

Gérard BÉAur.

Philippe Mroche, Le Plan Monnet, genèse et élaboration: 1941-1947. Paris, Publications de la Sorbonne, 1987. $16 \times 24,323$ p. ("France $X^{e} X^{e}-X^{e}$ ").

C'est à partir d'une thèse de troisième cycle, que l'auteur a conçu ce livre sur les origines et l'élaboration du Plan de modernisation et d'équipement, dont Jean Monnet remit au général De Gaulle en décembre 1945 les propositions. Moderniser la France en la reconstruisant, le faire avec tous les Français, au moyen de l'innovation stimulante que sont les commissions de modernisation où fonctionnaires, représentants du monde de l'entreprise et des syndicats peuvent débattre des voies de la modemisation : la personnalité de Jean Monnet n'est pas négligeable, qui découvre en 1945 le lent et régulier déclin d'un pays, dont la cause profonde serait le manque d'esprit d'entreprise.

Le Plan proposé en 1945 est élaboré en 1946 et adopté en 1947, et cette chronologie pose le premier problème des héritages et des origines du Plan. L'élaboration ne peut être dissociée des relations franco-américaines, dans la mesure où la France affaiblie est dépendante des États-Unis, et où Jean Monnet a des liens anciens et forts avec les mondes politique et des affaires américains. Enfin, le rôle des hommes, groupes sociaux et forces politiques qui ont, en France, permis la réflexion et la décision constitue le troisième axe problématique de l'ouvrage.

Les origines du Plan sont antérieurs à la Libération et à la guerre même. Jean

15. Jacques CAPDEVIELle, Le Fétichisme du patrimoine. Essai sur un fondement de la classe moyenne, Paris, Presses de la Fondation nationale des sciences politiques, 1986.

16. Etude de Michel Mouillart (Secteur logement du Centre d'études et de recherches sur la ville et l'espace), diffusée par le ministère de l'Equipement et du Logement.

17. Je pense par exemple au livre de Michel LesCuRE, Les Banques, l'Etat et le marché immobilier en France d l'époque contemporaine, 1820-1940, Paris, Éd. de l'École des hautes études en sciences sociales, 1982. 
Coutrot proclame en janvier 1938 «... la nécessité psychologique d'un plan » en précisant qu'il importe «... d'annoncer solennellement un perfectionnement de l'organisme gouvernemental [...]. Il serait vraisemblablement possible d'obtenir sur ce programme la collaboration de la C.G.T. et de la C.G.P.F. dont les représentants autorisés travaillent déjà côte à côte au sein du Centre national d'organisation scientifique du travail ". Le régime né de la défaite de 1940 s'inscrit en continuité des réflexions de l'avant-guerre. Le Comité d'études pour la France a rédigé cinquante-trois rapports en 1941 et 1942, dont l'un porte sur « l'organisation de la production industrielle " et comporte des objectifs de modernisation.

La composition de ce Comité de cinquante-neuf membres rassemble des personnalités de Vichy telles que F. Lehideux, A. Detoeuf ou Bichelonne, d'autres qui jouent un rôle dans le plan Monnet comme A. Sauvy. La création de la Délégation générale à l'équipement national (D.G.E.N.) dont le secrétaire général est F. Lehideux en 1941, sous le second gouvernement de l'amiral Darlan, permet de dégager trois objectifs économiques et sociaux dans le cadre d'un plan décennal : l'autarcie obtenue en partie grâce à l'Empire, le plein emploi (dans une conjoncture de chômage) et la régularité de la vie économique en redéfinissant le rôle de l'État. Ce dernier point, qui rencontre les objectifs du plan Monnet, permet d'insister sur le rôle de régulation contra-cyclique des pouvoirs publics. Au-delà d'une vision passablement passéiste de l'économie, l'organisation même de la D.G.E.N. en tant que maître-d'cuvre du plan et de la modernisation doit être soulignée, même si elle se heurta à de trop nombreux obstacles.

Obstacles dont le plus déterminant fut sans doute le temps : la rédaction du Plan est achevée le 15 mai 1944 et la tranche de démarrage n'est que prévue. Cependant Pierre Mendès France s'est inspiré de ces prévisions, comme de celles de Londres et d'Alger dans des domaines de réflexion fort parallèles à ceux de Vichy, illustrés par $G$. Boris et $R^{\prime}$ Courtin. Les mouvements politiques et des groupes sociaux ont d'ailleurs à la Libération leur idée d'un plan : les communistes entendent ainsi entreprendre et réaliser la Révolution avant de planifier l'économie du pays. Conflits idéologiques auxquels s'ajoutent des problèmes de subordination dans la conception et la décision : le directeur du Plan se heurte aux différents ministres de l'Économie nationale, pour lesquels le Plan rentre dans leurs attributions, d'après l'ordonnance du 23 novembre 1944; ce ministère se heurte aux autres départements ministériels concernés par le Plan.

C'est d'autant plus vrai qu'il s'agit, ainsi que le souligne P. Mendès France, d'un gouvernement d'Union nationale, composé de membres « ... réunis pour une grande œuvre commune, pour libérer la France d'abord, poursuivre la guerre jusqu'à la victoire ensuite ". Et il insiste pour que le chef du gouvernement soit l'arbitre. On sait qu'il le fut quant aux projets de plan conçus par $P$. Mendès France et auxquels s'opposaient aussi bien les communistes que R. Dautry ou R. Pléven. La démission de Pierre Mendès France ne signifia pas l'échec du Plan, mais un constat de désaccords quant à la conception même de la planification.

Le libéral $R$. Pléven s'attache d'abord à réorganiser le ministère de l'Économie nationale, et c'est à $R$. Dautry, ministre de la Reconstruction et de l'Urbanisme, qu'est confiée la responsabilité du Plan. C'est dans ce contexte que se place l'action de Jean Monnet. Homme de terrain et de réflexion, il négocie avec les 
États-Unis entre 1943 et 1945 la reconstruction française par l'intermédiaire du prêt-bail. C'est lui qui négocie le décret du 3 janvier instituant un Commissariat général au plan, dont il devient le premier titulaire. Dès son entrée en fonction, il doit résister à diverses tentatives de ministères qui entendent mettre la main sur ce nouvel organisme, finalement rattaché à la Présidence du Conseil.

Les hommes qui entourent $J$. Monnet sont peu nombreux, issus pour la plupart des grands corps - bien que J. Monnet préférât les industriels expérimentés. On y relève des noms connus : E. Hirsch, F. Gaillard, R. Marjolin, A. Sauvy, J. Fourastié, P. Delouvrier, S. Nora et d'autres encore. Il faut y voir là la promotion d'une nouvelle génération de décideurs. Au-delà des pouvoirs réglementaires et administratifs, la personnalité de J. Monnet, empiriste à l'anglosaxonne, permet de définir les éléments de travail. Le Conseil national du plan est crée le 8 mars 1947 et réunit des industriels, des syndicalistes ouvriers, et des agriculteurs. Entre mars 1946 et la fin 1947 sont mises en place vingt-quatre commissions (on en trouvera la liste chronologique p. 108).

Philippe Mioche insiste sur la nécessaire imbrication qui existe entre le plan Monnet et les négociations franco-américaines, notamment celles du printemps 1946 dites Blum-Byrnes : les États-Unis ont influé sur la reconstruction de la France, mais l'action de J. Monnet a influé sur la politique française des ÉtatsUnis, malgré le regard critique de l'économiste et statisticien $\mathbf{R}$. Nathan sur le Plan. Tout compte fait, il a contribué à donner à la France une avance relative parmi les nations européennes.

Le plan a été adopté en janvier 1947. Sa durée n'est pas précisée ; on s'accordait à la fin de l'année précédente sur une année pour d'immédiates décisions, et trois ans pour la réalisation des prévisions. Pour certains secteurs, l'échéance est plus longue. Au cours de l'année 1947, le plan devenu plus sélectif - définition des priorités, programme d'investissements - apparait comme un efficace outil de la politique économique et comme ayant contribué à créer les conditions de la croissance. Devenu symbole du redressement national, le plan Monnet le fut d'abord par sa réception aux différentes étapes de son élaboration. Participation active de la C.G.T., de la C.G.A., position prudente du C.N.P.F.; apparente unanimité des trois grands partis politiques qui masque des idées fort différentes sur la planification. Quant à l'opinion publique, elle paraît soit détachée, soit mal informée; l'année 1947 offre, par ailleurs, divers autres sujets de préoccupation.

La troisième partie du livre est consacrée aux commissions de modernisation du plan Monnet et à leur travail. Lieu privilégié d'un nouvel état d'esprit cher à J. Monnet, ces commissions ont présenté des bilans divers : échec de la commission de modernisation de la machine-outil - qui demeure jusqu'à ce jour un des points faibles du secteur secondaire de l'économie française -, résultats mitigés de la commission de modernisation de la sidérurgie, succès de la commission de modernisation des carburants.

$\mathrm{Au}$ terme de cette étude de la genèse et de l'élaboration du plan Monnet, l'auteur conclut à une indéniable réussite de J. Monnet et de ses collaborateurs. Réussite, parce qu'ils ont su construire un "triptyque méthodologique : la modestie du propos, la concision des objectifs et la clarté énoncée des enjeux ". Réussite, parce qu'ils ont fondé leur tâche sur un pari, qui consistait à accepter 
une dépendance financière à court terme pour faire recouvrer à la France, à long terme, sa puissance économique. Ni dirigiste, ni libéral, ce point d'équilibre rompu au seuil des années cinquante a néanmoins permis l'expansion subséquente des années 1960.

Tristan LECOQ.

\section{SOCIETES ET SCIENCES SOCIALES}

" Lèpre et société au Moyen Âge ", Sources. Travaux historiques, 13, 1988.

Ce numéro de la revue est presque exclusivement consacré à la lèpre au Moyen Âge. Sous la signature de F.-O. Touati, l'article liminaire « Histoire des maladies, histoire totale ", fait l'inventaire des exigences qui permettent d'appréhender le phénomène pathologique dans son entier. Ce souci de formuler des principes conduit l'auteur à emprunter largement aux historiens contemporains (la bibliographie sur le sujet est d'ailleurs exemplaire), afin d'établir une approche du faisceau de " discours ", constitutif de l'objet de la recherche historique : discours médical, " scientifique, juridique, politique, littéraire, moral, théologique, hagiographique et même historiographique ". Plus que tout autre maladie, la lèpre a conditionné son historiographie ; il s'agit donc de proposer une vision décapée, qui exige une chronologie fine de la maladie et la prise en compte de la dynamique de la société, qui se manifeste par l'exclusion. Trois secteurs semblent offrir un champ privilégié à cet examen approfondi : l'écart constaté entre une réalité nosologique apparemment bien définie et les constants glissements sémantiques à l'égard du référent opérés par la pensée savante et théologique, l'écart constaté entre la réalité de l'endémie et les réalisations hospitalières, l'analogie entre l'État monastique et la situation du lépreux exclu de la communauté.

L'article d'Isabelle Cochelin, "Bourgeoisie et léproserie de la principauté de Liège ", s'inscrit dans ce programme et évoque l'histoire de deux ladreries prises en charge, moins d'un siècle après leur apparition par les magistrats urbains. Plus que le pouvoir rédempteur de la charité, c'est le désir des bourgeois, soucieux de jouer un rôle actif dans l'administration des villes qui explique ce transfert. Bruno Tabuteau, sous le titre "Combien de lépreux au Moyen Âge? ", s'efforce à partir des exemples de Rouen et de Bellemcombre au XIII' siècle, de préciser le taux 